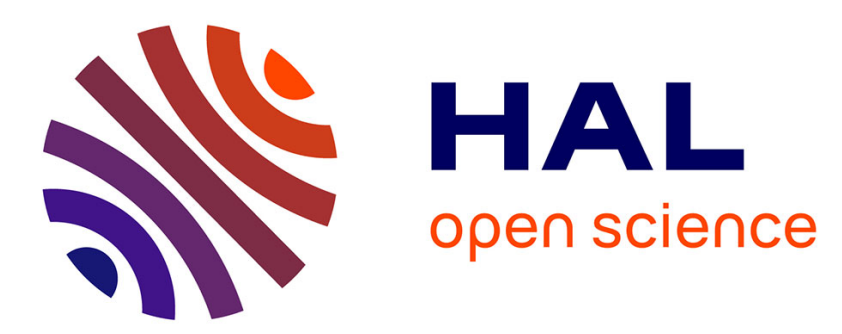

\title{
Adaptive Backstepping and MEMS Force Sensor for an MRI-Guided Microrobot in the Vasculature
}

Laurent Arcèse, Matthieu Fruchard, Felix Beyeler, Antoine Ferreira, B.-J.

Nelson

\section{- To cite this version:}

Laurent Arcèse, Matthieu Fruchard, Felix Beyeler, Antoine Ferreira, B.-J. Nelson. Adaptive Backstepping and MEMS Force Sensor for an MRI-Guided Microrobot in the Vasculature. 2011 IEEE International Conference on Robotics and Automatics, May 2011, Shangaï, China. pp 4121-4126. hal-00656004

\section{HAL Id: hal-00656004 https://hal.science/hal-00656004}

Submitted on 3 Jan 2012

HAL is a multi-disciplinary open access archive for the deposit and dissemination of scientific research documents, whether they are published or not. The documents may come from teaching and research institutions in France or abroad, or from public or private research centers.
L'archive ouverte pluridisciplinaire HAL, est destinée au dépôt et à la diffusion de documents scientifiques de niveau recherche, publiés ou non, émanant des établissements d'enseignement et de recherche français ou étrangers, des laboratoires publics ou privés. 


\title{
Adaptive backstepping and MEMS force sensor for an MRI-guided microrobot in the vasculature
}

\author{
Laurent Arcese, Matthieu Fruchard, Felix Beyeler, Antoine Ferreira, Bradley J. Nelson
}

\begin{abstract}
A microrobot consisting of a polymer binded aggregate of ferromagnetic particles is controlled using a Magnetic Resonance Imaging (MRI) device in order to achieve targeted therapy. The primary contribution of this paper is the design of an adaptive backstepping controller coupled with a high gain observer based on a nonlinear model of a microrobot in a blood vessel. This work is motivated by the difficulty in accurately determining many biological parameters, which can result in parametric uncertainties to which model-based approaches are highly sensitive. We show that the most sensitive parameter, magnetization of the microrobot, can be measured using a Micro-Electro-Mechanical Systems (MEMS) force sensor, while the second one, the dielectric constant of blood, can be estimated on line. The efficacy of this approach is illustrated by simulation results.
\end{abstract}

\section{INTRODUCTION}

Minimally invasive cancer therapy is currently an active research area since these techniques can, both, reach remote places without invasive surgery and reduce drug or radiation dosage. The techniques result in reduced side effects, better therapeutic efficacy, and are safer overall. The drawbacks of classical therapeutic approaches can be avoided by targeted chemotherapy which relies on selective delivery using either drugs linked to antibodies specific for tumor-associated antigenes [1], or drugs carried by autonomous untethered microrobots controlled so as to reach the tumor. However, it have proven difficult to embed actuators sufficiently powerful to propel such robots in the cardiovascular system. Most swimming approaches, consequently, rely upon magnetic fields to wirelessly transmit power to the microrobot [2], [3], [4]. A recent magnetic actuation technique particularly well-suited for medical purposes has been described by Martel [5]. The authors developed a "bead pulling" technique through the use of the gradient coils of clinical MRI devices to provide propelling power. The relevance of using clinical MRI devices is twofold: the machines are wide spread in hospitals, and their imaging ability yields fine observation of the robot's position in the cardiovascular system.

L. Arcese and M. Fruchard are with the Institut PRISME UPRES 4229 , IUT de Bourges, 63 Av de Lattre de Tassigny, 18020, Bourges cedex, France, laurent.arcese@bourges.univ-orleans.fr matthieu. fruchard@bourges.univ-orleans.fr

F. Beyeler is with FemtoTools GmbH, ETHZ-IRIS, Tannenstrasse 3, CH8092, Zurich, Switzerland, felix.beyelerefemtotools.com

A. Ferreira is with the Institut PRISME UPRES 4229, ENSI de Bourges, 88 Bd Lahitolle, 18000, Bourges, France antoine.ferreiradensi-bourges.fr

B.J. Nelson is with Institute of Robotics and Intelligent Systems, ETH Zurich, Tannenstrasse 3, CH-8092, Zurich, Switzerland, bnelson@ethz.ch
To deliver drugs close to the tumor, navigating in smaller vessels while avoiding embolization hazards suggests using robots down to one hundred micrometers [6]. However, clinical MRI gradients coils are not strong enough to steer such nanorobots in larger vessels. Furthermore, hydrodynamic wall effects show that a partial vessel occlusion by the robot results in an optimal ratio between the robot's and the vessel's radius [5], [7]. An obvious way to overcome MRI limitations is to decrease the radius of the robot as it enters smaller vessels, e.g. with a microrobot made of a degradable polymer binded aggregate of magnetic particles.

In this paper, we first develop a mechanics model (Section II) that includes wall effects, wall interactions and the nonNewtonian behavior of blood. Although this forces balance results in a highly nonlinear model, the great majority of related literature synthesizes control laws using linear tools, and there is little consideration concerning observation issues. Hence, instabilities and sensitivity to noise or to unmodeled dynamics, reported for instance in [8] with a PID controller, is important to consider. In [9], we developed a backstepping controller that proves stable but sensitive to some small physiological parametric errors. In this paper, we show that an adaptive backstepping control approach (Section III) greatly improves the quality and robustness of the tracking along a reference trajectory. As the MRI imager only measures the robot's position, we use a high gain observer to reconstruct the microrobot's velocity, which is required to implement the control law. In Section IV, we show that the use of a MEMS-based force sensor is effective in precisely measuring the magnetization of the microrobot, and that simulation illustrates the benefits of using an adaptive controller-observer pair to improve the robustness of the approach with respect to physiologically uncertain parameters. Finally, a conclusion and future prospects are discussed.

\section{MODELING}

The therapeutic microrobot is a polymer binded aggregate of ferromagnetic particles that is immersed in a blood vessel. We briefly present forces acting on such a robot (for a more detailed discussion on the modeling, see [9]).

The translational motion of the robot is expressed by:

$$
m \frac{d \vec{v}}{d t}=\vec{F}_{m}+\vec{W}_{a}+\vec{F}_{d}+\vec{F}_{c}+\vec{F}_{v d w}+\vec{F}_{e}
$$

where $\vec{v}$ is the translational velocity of the robot and $m$ its mass. In the remainder of the paper, we assume that the 
orientation of the robot does not change due to magnetic torque that constantly align the magnetization of the robot along the external MRI field $\left(B_{0}=3 T\right)$.

\section{A. Volumetric forces}

The gradient coils of the MRI system provide magnetic gradients that produce a magnetic force $F_{m}$ on the robot:

$$
\vec{F}_{m}=\tau_{m} V(\vec{M} \cdot \nabla) \vec{B}
$$

where $V$ is the robot's total volume, $\tau_{m}=\frac{V_{m}}{V}$ with $V_{m}$ the ferromagnetic volume, $\vec{M}$ is the magnetization of the material, $\mu_{0}$ is the permeability of free space and $\nabla \vec{B}$ is the magnetic field gradient. Note that $B_{0}$ is sufficient to ensure a saturation magnetization $M_{\text {sat }}$.

Apparent weight (combined action of weight and buoyancy) also acts on the spherical robot:

$$
\vec{W}_{a}=V\left(\rho-\rho_{f}\right) \vec{g}
$$

where $\rho=\tau_{m} \rho_{m}+\left(1-\tau_{m}\right) \rho_{\text {poly }}$ with $\rho_{m}$ and $\rho_{\text {poly }}$ the density of the magnetic material and polymer, respectively.

\section{B. Hydrodynamic drag force}

The hydrodynamic drag force $\vec{F}_{d}$ exerted on a spherical body is expressed as:

$$
\vec{F}_{d}=-\frac{1}{2} \rho_{f}\left[\frac{\left\|\left(\vec{v}-\vec{v}_{f}\right)\right\|}{\beta}\right]^{2} A C_{d} \frac{\left(\vec{v}-\vec{v}_{f}\right)}{\left\|\left(\vec{v}-\vec{v}_{f}\right)\right\|}
$$

where $\vec{v}-\vec{v}_{f}$ denotes the relative velocity of the robot with respect to the fluid, $A$ is the projected area of the sphere, $\rho_{f}$ is the density of the fluid. $\beta$, a dimensionless ratio related to a wall effect caused by the vessel's occlusion by the robot, and $C_{d}$, the drag coefficient, are discussed in [9]. In the case of blood, which exhibits a non-newtonian behavior, the fluid's viscosity $\eta$ is a function of the vessel's diameter and hematocrit rate $h_{d}$ according to empirical relations given in [10]. Wall effects on the fluid in a vessel traditionally result in a parabolic profile of blood flow (see Figure 1).

\section{Contact force}

The contact force is expressed by a modified Hertzian contact law [11] under the simplifying assumption of no friction between the robot and the wall during impact:

$$
\left\{\begin{array}{l}
\vec{F}_{c}=k \delta^{3 / 2} \cdot \vec{n} \quad: \text { loading } \\
\vec{F}_{c}=F_{m}\left(\frac{\delta-\delta_{0}}{\delta_{m}-\delta_{0}}\right)^{q} \cdot \vec{n} \quad: \text { unloading }
\end{array}\right.
$$

where $\delta$ is the elastic deformation of the wall at the contact point, $\vec{n}$ the normal unit vector pointing from the robot to the contact surface and $k$ the stiffness. $E_{p}$ and $E_{w}$ are the Young's modulus of robot and wall, and $\sigma_{p}$ is the Poisson's ratios of robot. $F_{m}$ and $\delta_{m}$ are the maximum contact force and deformation of the wall reached at the impact. $\delta_{0}$ is the permanent wall's deformation from loading/unloading cycle and the exponent $q$ varies between 1.5 and 2.5.

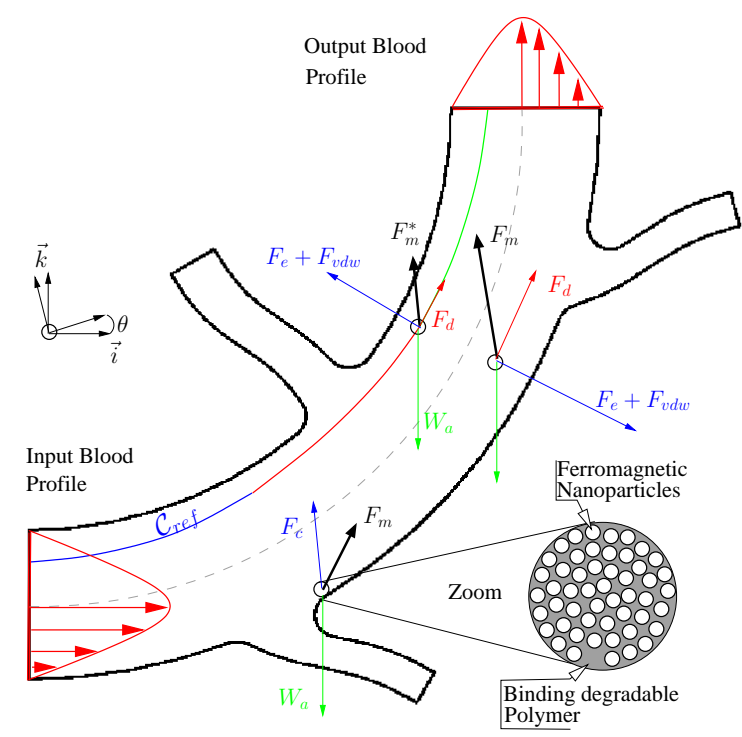

Fig. 1. Schematic of a blood vessel with minor bifurcations.

\section{Van der Waals and electrostatic forces}

The Van der Waals potential and the electrostatic force between the spherical robot and the wall considered as an uncharged surface are given by [12], [13]:

$$
\left\{\begin{array}{l}
\vec{V}_{v d w}=-\frac{A_{h}}{6}\left(\frac{1}{H}+\frac{1}{2+H}+\ln \frac{H}{2+H}\right) \vec{n} \\
\vec{F}_{e}=\frac{q^{2}}{4 \pi \epsilon \epsilon_{0}(r+h)^{2}} \vec{n}
\end{array}\right.
$$

where $A_{h}$ is the Hamaker constant, $H=h / r$ is normalized separation $h$ between the robot and the wall, $q(\mu C)=$ $120 \pi r^{2}(100 r)^{-0.3}$ denotes the robot's charge [14], $\epsilon$ the dielectric density of the medium in which the interaction occurs and $\epsilon_{0}$ the vacuum permittivity.

\section{CONTROL DESIGN}

\section{A. State space representation}

Let $(x, z)$ denote the position of the robot in the vessel with respect to a given frame $\mathcal{F}(O, \vec{i}, \vec{k})$. The state model is established from the differential equation (1) defining the robot's dynamic behavior, projected on the $\vec{i}$ and $\vec{k}$ axes:

$$
\left\{\begin{array}{l}
m \ddot{x}=F_{m_{x}}+F_{d_{x}}+F_{c_{x}}+F_{v d w_{x}}+F_{e_{x}} \\
m \ddot{z}=F_{m_{z}}+F_{d_{z}}+W_{a}+F_{c_{z}}+F_{v d w_{z}}+F_{e_{z}}
\end{array}\right.
$$

where indexes $x$ and $z$ represent projections onto $\vec{i}(\vec{k})$.

Let $x_{1}, x_{2},\left(x_{3}, x_{4}\right)$ denote, respectively, the particle's position and velocity along the $\vec{i}$ axis (respectively along $\vec{k}$ axis). Assuming that positions $x_{1}$ and $x_{3}$ can be measured by the MRI imaging system, let $y$ denotes the measured state. Using expressions of forces given by (2), (3), (4), (6), and the projection of the local frame along the geometry of the 
vessel, (7) can be written in the form:

$$
(\mathcal{S})\left\{\begin{array}{l}
\dot{x}_{1}=x_{2} \\
\dot{x}_{2}=f_{2}\left(x_{1}, x_{2}, x_{3}\right)+a u_{1} \\
\dot{x}_{3}=x_{4} \\
\dot{x}_{4}=f_{4}\left(x_{1}, x_{3}, x_{4}\right)+a u_{2} \\
y=\left(x_{1}, x_{3}\right)^{T}
\end{array}\right.
$$

where control inputs $u_{1}=\nabla B_{x}$ and $u_{2}=\nabla B_{z}$ are the magnetic gradients, parameter $a=\frac{\tau_{m} M}{\rho}$, and $f_{i}$ are highly nonlinear functions of the full state.

\section{B. Adaptive backstepping}

From the previous force balance, an optimal trajectory is defined so as to minimize the control efforts. We first define optimal points so as to minimize the control efforts (i.e. the magnetic force $F_{m}$ required), then link them using B-splines in order to obtain a $\mathcal{C}^{2}$ reference trajectory.

An adaptive nonlinear control [15] based on a backstepping approach, for system $(\mathcal{S})$, can be developed. The objective of the adaptive backstepping controller is twofold. Since the blood's dielectric density $\varepsilon$ is assumed to be unknown, it must define both an update law for the estimated parameter and control inputs for the controlled system. The update law must ensure convergence of the estimated parameter to its real value, while the control inputs must stabilize the tracking error between the actual and reference trajectory to zero. Since this design requires a triangular form for the control system, we define the following change of variables:

$$
\left\{X=\left(\begin{array}{l}
x_{1} \\
x_{3}
\end{array}\right) ; \quad Z=\left(\begin{array}{l}
x_{2} \\
x_{4}
\end{array}\right) ; \quad U=a\left(\begin{array}{l}
u_{1} \\
u_{2}
\end{array}\right)\right.
$$

From (8) and (9), we obtain a new system in a triangular form:

$$
\left(\mathcal{S}^{\prime}\right)\left\{\begin{array}{l}
\dot{X}=Z \\
\dot{Z}=F_{0}(X, Z)+\varphi(X) \theta+U \\
Y=X
\end{array}\right.
$$

where $\varphi(X)$ is the electrostatic force without the parameter $\varepsilon, \theta$ the vector of unknown constant parameter and with:

$$
F_{0}(X, Z)=\left(\begin{array}{l}
F_{d_{x}}+F_{c_{x}}+F_{v d w_{x}} \\
F_{d_{z}}+W_{a}+F_{c_{z}}+F_{v d w_{z}}
\end{array}\right)
$$

Using the change of variables given in (9), we construct the control law in two steps.

\section{Step 1: Let}

$$
\left\{\begin{array}{l}
\tilde{X}=X-X_{r} \\
\tilde{Z}=Z-Z_{r}-\alpha
\end{array}\right.
$$

denote the position and velocity error, respectively. $X_{r}$, $\dot{X}_{r}=Z_{r}$ and $\ddot{X}_{r}$ are the desired reference trajectory, velocity and acceleration, respectively. $\alpha$ is a stabilizing function.
A control Lyapunov function (CLF) candidate is:

$$
V_{1}=\frac{1}{2} \tilde{X}^{T} \tilde{X}+\frac{1}{2}(\hat{\theta}-\theta)^{T} \Gamma^{-1}(\hat{\theta}-\theta) \quad \geq 0
$$

where $\hat{\theta}$ is the estimated parameter and $\Gamma$ a positive-definite matrix.

Calculating the derivative of $V_{1}$ along system (10), we obtain:

$$
\dot{V}_{1}=\tilde{X}^{T}(\tilde{Z}+\alpha)+(\hat{\theta}-\theta)^{T} \Gamma^{-1} \dot{\hat{\theta}}
$$

Setting $\alpha=-k_{1} \tilde{X}$, we obtain:

$$
\dot{V}_{1}=-k_{1} \tilde{X}^{T} \tilde{X}+\tilde{X}^{T} \tilde{Z}+(\hat{\theta}-\theta)^{T} \Gamma^{-1} \dot{\hat{\theta}}
$$

The second term $\tilde{X}^{T} \tilde{Z}$ will be cancelled at the next step. One can notice from the previous expressions that:

$$
\dot{\tilde{X}}=\dot{X}-\dot{X}_{r}=\tilde{Z}+\alpha=\tilde{Z}-k_{1} \tilde{X}
$$

Step 2: From (12) and (16), we have:

$$
\dot{\tilde{Z}}=\underbrace{F_{0}+\varphi \theta+U}_{\dot{Z}}-\dot{Z}_{r} \underbrace{+k_{1}\left(\tilde{Z}-k_{1} \tilde{X}\right)}_{-\dot{\alpha}}
$$

In this step, the CLF is given by:

$$
V_{2}=V_{1}+\frac{1}{2} \tilde{Z}^{T} \tilde{Z} \quad \geq 0
$$

The derivative of $V_{2}$ along system (10) is expressed as:

$$
\begin{aligned}
\dot{V}_{2}= & \dot{V}_{1}+\tilde{Z}^{T}\left[F_{0}+\varphi \theta+U+k_{1} \tilde{Z}-k_{1}^{2} \tilde{X}-\dot{Z}_{r}\right] \\
= & -k_{1} \tilde{X}^{T} \tilde{X}+\tilde{Z}^{T}\left[\left(1-k_{1}^{2}\right) \tilde{X}+k_{1} \tilde{Z}-\dot{Z}_{r}+F_{0}\right. \\
& +\varphi \theta+U]+(\hat{\theta}-\theta)^{T} \Gamma^{-1} \dot{\hat{\theta}}
\end{aligned}
$$

Since we have $\theta=\hat{\theta}+(\theta-\hat{\theta})$, we obtain:

$$
\begin{aligned}
\dot{V}_{2}= & -k_{1} \tilde{X}^{T} \tilde{X}+\tilde{Z}^{T}\left[\left(1-k_{1}^{2}\right) \tilde{X}+k_{1} \tilde{Z}-\dot{Z}_{r}+F_{0}\right. \\
& +\varphi \hat{\theta}+U]+(\hat{\theta}-\theta)^{T} \Gamma^{-1}\left(\dot{\hat{\theta}}-\Gamma \varphi^{T} \tilde{Z}\right)
\end{aligned}
$$

To cancel the last term in (20), we set:

$$
\dot{\hat{\theta}}=\Gamma \varphi^{T} \tilde{Z}
$$

and to ensure $\dot{V}_{2}$ is negative definite, we set:

$$
\left(1-k_{1}^{2}\right) \tilde{X}+k_{1} \tilde{Z}-\dot{Z}_{r}+F_{0}+\varphi \hat{\theta}+U=-k_{2} \tilde{Z}
$$

To summarize, from (16), (21) and (22), the control law $U$ and the update law for the parameter estimate $\hat{\theta}$ can be expressed as:

$$
\left\{\begin{aligned}
U= & \ddot{X}_{r}-\left(k_{1}+k_{2}\right)\left(Z-\dot{X}_{r}\right)-\left(1+k_{1} k_{2}\right)\left(X-X_{r}\right) \\
& -F_{0}-\varphi \hat{\theta} \\
\dot{\hat{\theta}}= & \Gamma \varphi^{T}\left[Z-\dot{X}_{r}+k_{1}\left(X-X_{r}\right)\right]
\end{aligned}\right.
$$




\section{High gain observer}

The adaptive nonlinear control (23) requires the knowledge of the unmeasured robot's velocity $Z$ in addition to its position, which is measured by the MRI device. The extended Kalman filter is an observer commonly used to estimate the full state, but the convergence of this observer is not guaranteed. The high gain observer [16] is of particular interest for our application since, if the state's functions are locally Lipschitzian and the system has the canonical form given in (10), we can guarantee the observer's convergence. The high gain observer is, thus, given by:

$$
\left\{\begin{array}{l}
\dot{\hat{X}}=\hat{Z}+L G_{x}(\hat{X}-Y) \\
\dot{\hat{Z}}=F_{0}(\hat{X}, \hat{Z})+\varphi(\hat{X}) \theta+U+L^{2} G_{z}(\hat{X}-Y)
\end{array}\right.
$$

where $L$ is the high gain, $G_{x}=\left(\begin{array}{cc}g_{1} & 0 \\ 0 & g_{3}\end{array}\right)$ and $G_{z}=\left(\begin{array}{cc}g_{2} & 0 \\ 0 & g_{4}\end{array}\right)$ are defined from the Hurwitz matrix $H_{u}$ :

$$
H_{u}=\left(\begin{array}{cccc}
g_{1} & 0 & 1 & 0 \\
0 & g_{3} & 0 & 1 \\
g_{2} & 0 & 0 & 0 \\
0 & g_{4} & 0 & 0
\end{array}\right)
$$

\section{RESULTS}

\section{A. Magnetization measurement}

The magnetization of the $\mathrm{NdFeB} \mathrm{N} 35$ microrobot is one of the most sensitive parameters, as it appears in factor of the control inputs. To experimentally obtain quantitative magnetization values, force measurements have been performed inside a known magnetic field using a FemtoTools FT-S270 capacitive MEMS force sensor. The maximum force range of the MEMS sensor is $\pm 2000 \mu N$ and the resolution is in the sub- $\mu N$ range. First, the microrobot is glued to the MEMS sensor probe (see Figure 2 ). In order to get meaningful results, the direction of the magnetization relative to the sensor probe must be known. Unfortunately, magnetization cannot be directly seen. To overcome this issue the microrobot was aligned prior to gluing as shown in figure 3. It is first put onto a flat surface made of non-magnetic material (PMMA). Then, a strong permanent magnet is placed underneath this spacer layer that automatically rotates the microrobot until it is aligned with the field lines of the underlying magnet.

For hard magnetic materials the magnetization does not depend on the externally applied field. The force generated by the $\mathrm{NdFeB} \mathrm{N} 35$ microrobot can be predicted by equation

TABLE I

FORCE AND MAGNETIZATION BY HARD-MAGNETIC MICROROBOT

\begin{tabular}{|c|c|c|}
\hline & $\begin{array}{c}\text { Maximum Force at } B=0.18 T \\
\text { and } \nabla B=25 T . m^{-1}\end{array}$ & Magnetization $M$ \\
\hline Microrobot 1 & $2020 \mu N$ & $1.23 \times 10^{6} A . m^{-1}$ \\
\hline Microrobot 2 & $2001 \mu N$ & $1.22 \times 10^{6} A . m^{-1}$ \\
\hline Microrobot 3 & $1890 \mu N$ & $1.16 \times 10^{6} A . m^{-1}$ \\
\hline
\end{tabular}

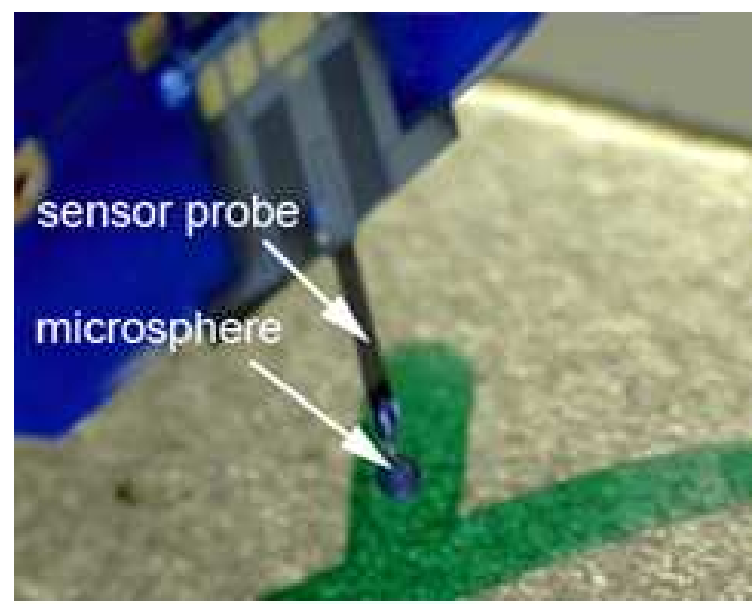

Fig. 2. Photograph of the microrobot glued to the sensor.

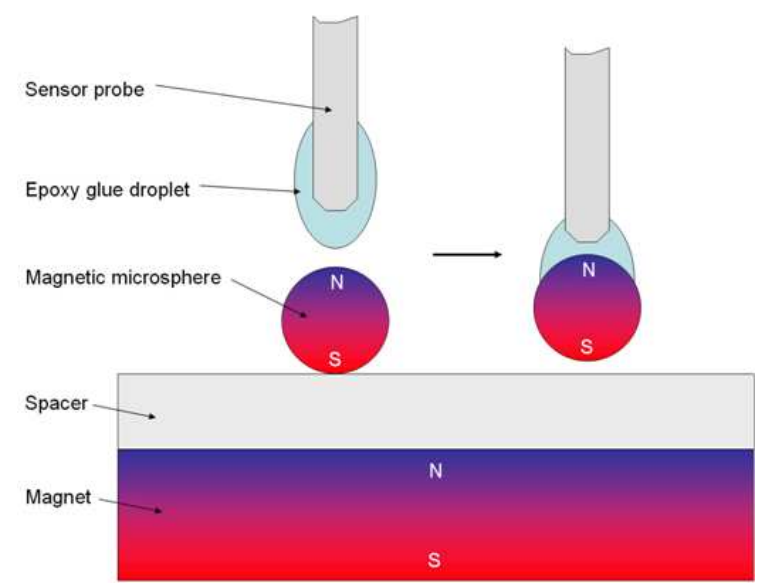

Fig. 3. Microrobot alignment and gluing to MEMS sensor probe tip.

(2). Table I shows the maximum force as well as the magnetization $M$ for the three samples that have been measured.

\section{B. Simulation}

Simulation is performed by taking into account the technical characteristics of recent clinical MRI devices. The magnetic field of $3 T$ is strong enough to assume that the particles reach their saturation magnetization. The magnetic field gradients are used to control the microrobot's motion in the human body. These MRI devices can provide a maximum magnetic field gradient of $80 \mathrm{mT} . \mathrm{m}^{-1}$. So, the applied control law $U_{a}(t)$ is corrected as follows so as not to exceed the capacity of the MRI system and to avoid damage:

$$
U_{a}(t)=\frac{U(t)}{k(t)} \quad \text { with } \quad k(t)=\max \left[1, \frac{U(t)}{U_{\text {sat }}}\right]
$$

The pulsating velocity of blood is modeled by an affine combination of a time-varying periodic flow with a spatial parabolic shape. To simplify the analytical expression, but with no loss of generality, we only consider the first terms in the time-varying Fourier series of the physiological pulse. 

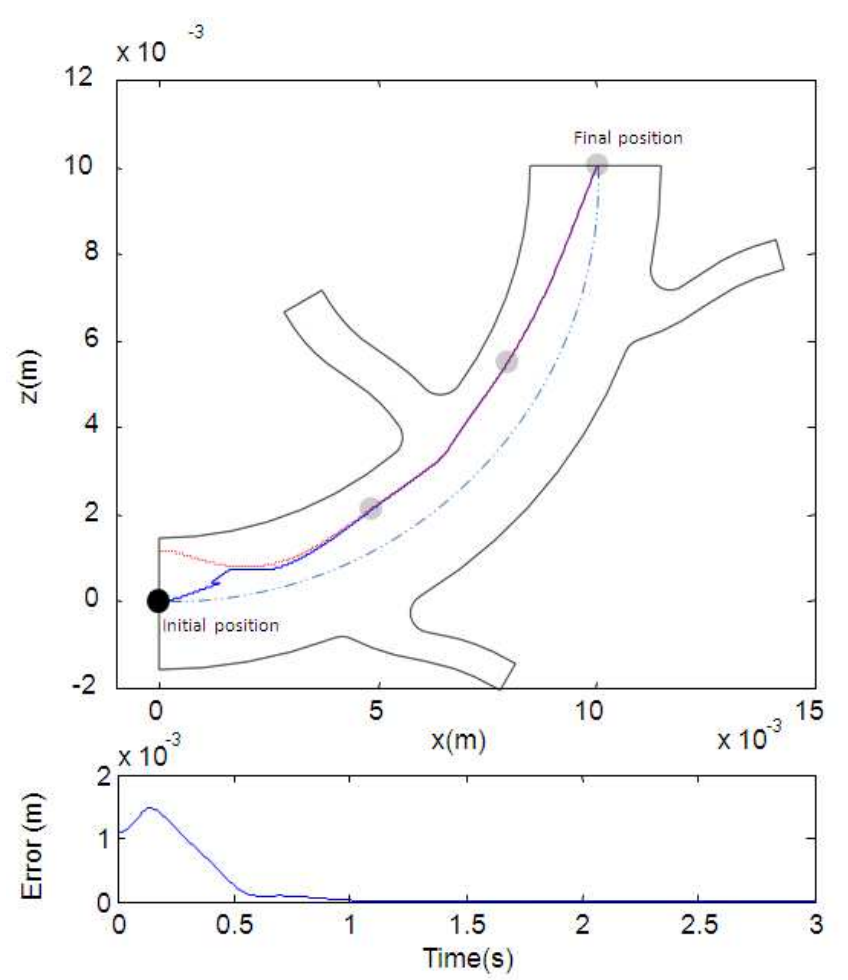

Fig. 4. (a) Reference (red dotted line) and simulated (blue solid line) trajectories. (b) Tracking error.

In the case of an artery, such an approximation leads to:

$v_{f}(t)=0.025\left(1+1.15 \sin \left(2 \pi t+\frac{\pi}{2}\right)\right) \times\left[1-\left(\frac{D / 2-h}{r}\right)^{2}\right]$

To fully take into account pulsatile flow in arteries caused by cardiac pumping, one must consider a periodic $10 \%$ deformation of the vessel's diameter $D(t)$, synchronized with the pulsative blood's velocity $v_{f}(t)$.

This simulation is performed assuming an error of $20 \%$ from the nominal blood's dielectric value. The motivation for estimating the uncertain parameter $\epsilon$ on-line is twofold. First, among the physiological parameters of the model, which are the most difficult to measure and are very variable from one patient to another, sensitivity is the highest for $\epsilon$, and, to a lesser extent, for $\eta$ and $A_{h}$. Second, adaptive backstepping cannot effectively deal with nonlinear parameters such as viscosity $\eta$.

The simulation demonstrates the relative stability of the proposed controller-observer. It shows that the parametric error affects neither the stability of the closed-loop system (Figure 4(a)), nor the convergence of the observer (Figure 5). Nevertheless, we note an important tracking error between $t=0 \mathrm{~s}$ and $t=1 \mathrm{~s}$. This phase is critical since the control inputs (Figure 7) reach the actuator saturation in the range $t \in\left[\begin{array}{ll}0 & 0.2\end{array}\right] \mathrm{s}$, and the parameter is not updated. Then, from $t=0.2 \mathrm{~s}$ to $t=1 \mathrm{~s}$, the estimated parameter is updated. The tracking error as well as the microrobot's velocity estimation error (Figure 5(b)) are affected by the
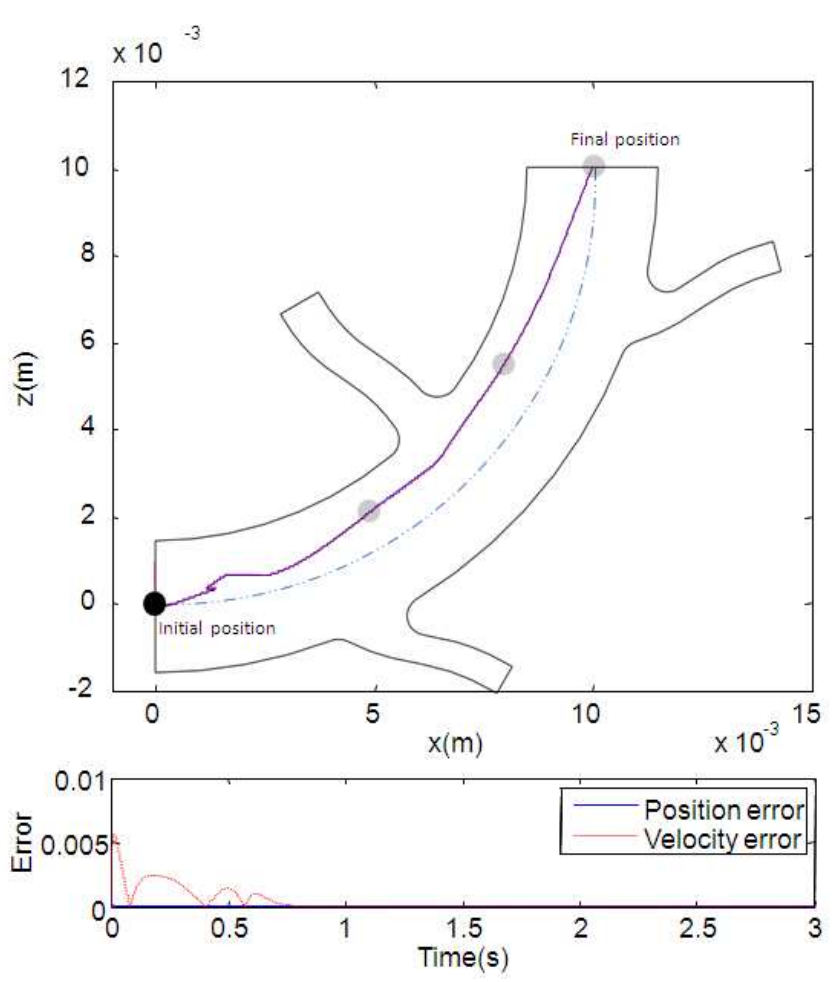

Fig. 5. (a) Simulated (blue solid line) and estimated (red dotted line) trajectories. (b) Position (blue solid line) and velocity (red dotted line) estimation error.

parametric error because the model-based controller and observer are quite sensitive to this uncertainty. However, as the update law gradually stabilizes the parametric error to zero (Figure 6), we observe a noticeable improvement of both tracking and estimation efficiency. The adaptive backstepping approach applied in this simulation thus fulfills two goals. During the transient phase, when estimated parameter has not yet converged, the controller/observer pair ensures a degraded but good tracking and estimation of velocity, thus demonstrating robustness to parametric uncertainties. After the estimated parameter has converged to its real value, the controller ensures an essentially perfect stabilization of the microrobot along the reference trajectory.

\section{CONCLUSION}

In this paper, we have developed an adaptive backstepping controller in order to perform on-line estimation of key physiological parameters for a therapeutic microrobot navigating in a blood vessel. This controller has been coupled with a high gain observer in order to reconstruct the full state needed to compute the control law. We have also described a method to measure the magnetization of the microrobot using a MEMS force sensor. The simulation results have shown the validity of our approach and the stability of the controller/observer pair. Comparison with our previous backstepping controller [9], where a similar parametric error induced a tracking error's mean value over $0.5 \mathrm{~mm}$, clearly illustrates the benefit of using adaptive backstepping. 
TABLE II

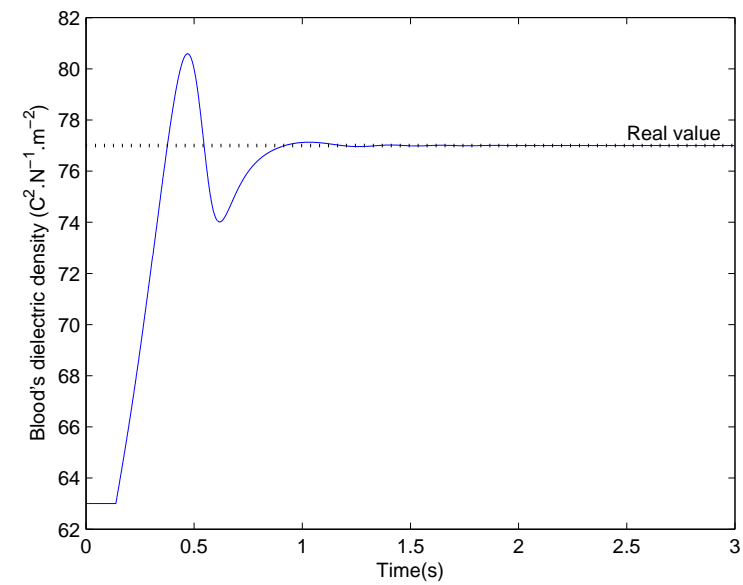

Fig. 6. Estimation of the blood's dielectric density $\epsilon$.

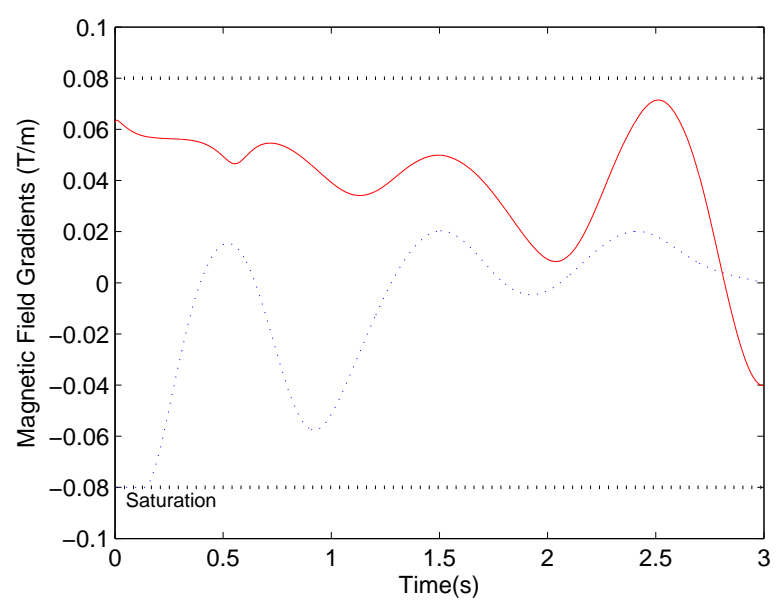

Fig. 7. Control input : magnetic field gradients on $\vec{i}$-axis (blue dotted line) and on $\vec{k}$-axis (red solid line).

The adaptive backstepping controller developed in this paper allows for the estimation of linear parameters, or those in which a change of a variable results in a linear case, in the model. The estimation of non-linearly varying parameters, such as blood viscosity, requires further study and remains an outstanding problem.

\section{ACKNOWLEDGMENTS}

This work was supported by European Union's 7th Framework Program and its research area ICT-2007.3.6 Micro/nanosystems under the project NANOMA (NanoActuactors and Nano-Sensors for Medical Applications).

\section{REFERENCES}

[1] E. Sievers et al., "Selective ablation of acute myeloid leukemia using antibody-targeted chemotherapy: a phase 1 study of an anti-cd33 calicheamicin immunoconjugate," Blood, vol. 97, no. 11, pp. 36783684, 1999.

[2] G. T. Gillies, R. C. Ritter, W. C. Broaddus, M. S. Grady, M. A. Howard, and R. G. McNeil, "Magnetic manipulation instrumentation for medical physics research," Rev. of Sci. Instruments, vol. 65, no. 3, March 1994.
SiMULATIONS DATA

\begin{tabular}{|c||c|c|}
\hline Plasma's viscosity & $\eta_{\text {plasma }}$ & $5 \times 10^{-3}[\mathrm{~Pa} . \mathrm{s}]$ \\
\hline Blood's density & $\rho_{f}$ & $1060\left[\mathrm{~kg} \cdot \mathrm{m}^{-3}\right]$ \\
\hline Robot's density & $\rho_{m}$ & $7500\left[\mathrm{~kg} \cdot \mathrm{m}^{-3}\right]$ \\
\hline Robot's radius & $r$ & $250[\mu \mathrm{m}]$ \\
\hline Robot's Young modulus & $E_{p}$ & $10^{9}[\mathrm{~Pa}]$ \\
\hline Wall's Young modulus & $E_{w}$ & $10^{6}[\mathrm{~Pa}]$ \\
\hline Robot's Poisson's ratio & $\sigma_{p}$ & 0.27 \\
\hline Permanent deformation & $\delta_{0}$ & $0[\mathrm{~m}]$ \\
\hline Vessel's diameter & $D$ & $3[\mathrm{~mm}]$ \\
\hline Polymer's density & $\rho_{\text {poly }}$ & $1500\left[\mathrm{~kg} \cdot \mathrm{m}^{-3}\right]$ \\
\hline Ferromagnetic ratio & $\tau_{m}$ & 0.8 \\
\hline Magnetization & $M$ & $1.23 \times 10^{6}\left[\mathrm{A.m} \mathrm{m}^{-1}\right]$ \\
\hline Hematocrit & $h_{d}$ & 0.45 \\
\hline Hamaker constant & $A_{h}$ & $4 \times 10^{-19}[\mathrm{~J}]$ \\
\hline Blood's dielectric density & $\varepsilon$ & $77\left[\mathrm{C}^{2} \cdot \mathrm{N}^{-1} \cdot \mathrm{m}^{-2}\right]$ \\
\hline Initial condition on $X, Z$ & $X_{0}$ & $(0,0)^{T}$ \\
\cline { 2 - 3 } & $Z_{0}$ & $(0,0)^{T}$ \\
\hline Initial condition on $\hat{X}, \hat{Z}$ & $\hat{X}_{0}$ & $(0,0)^{T}$ \\
\cline { 2 - 3 } & $\tilde{Z}_{0}$ & $(0.001,0)^{T}$ \\
\hline Inputs saturations & $U_{s a t}$ & $80\left[\mathrm{mT} \cdot \mathrm{m}^{-1}\right]$ \\
\hline Controller gains & $k_{1}, k_{2}$ & 7,14 \\
\hline Observer gains & $L$ & 19 \\
\cline { 2 - 3 } & $g_{1}, g_{2}, g_{3}, g_{4}$ & $-6,-13,-12,-4$ \\
\hline
\end{tabular}

[3] T. Honda, K. I. Arai, and K. Ishiyama, "Micro swimming mechanisms propelled by external magnetic fields," IEEE Trans. on Magnetics, vol. 32, no. 5, 1996.

[4] J. Abbott, Z. Nagy, F. Beyeler, and B. Nelson, "Robotics in the small: Part 1 microrobotics," IEEE Robotics and Automation Magazine, vol. 14, no. 2, pp. 92-103, June 2007.

[5] J.-B. Mathieu, G. Beaudoin, and S. Martel, "Method of propulsion of a ferromagnetic core in the cardiovascular system through magnetic gradients generated by an mri system," IEEE Transactions on Biomedical Engineering, vol. 53, no. 2, pp. 292-299, 2006.

[6] R. Bawa, "Nanoparticle-based therapeutics in humans : A survey," Nanotechnology Law \& Business, vol. 5:(2), pp. 135-155, 2008.

[7] P. Vartholomeos and C. Mavroidis, "Simulation platform for selfassembly structures in mri-based nanorobotic drug delivery systems," IEEE International Conference on Robotics and Automation, pp. 5594 - 5600, May 2010.

[8] S. Tamaz, R. Gourdeau, A. Chanu, J.-B. Mathieu, and S. Martel, "Real-time mri-based control of a ferromagnetic core for endovascular navigation," IEEE Trans. on Biomed. Eng., vol. 55, no. 7, July 2008.

[9] L. Arcese, A. Cherry, M. Fruchard, and A. Ferreira, "High gain observer for backstepping control of a mri-guided therapeutic microrobot in blood vessels," in IEEE International Conference on Biomedical Robotics and Biomechatronics, September 2010, to be published.

[10] A.-R. Pries, T.-W. Secomb, and P. Gaehtgens, "Biophysical aspects of blood flow in the microvasculature," Cardiovascular Research, vol. 32(4), pp. 654-667, 1996.

[11] I. Choi and C. Lim, "Low-velocity impact analysis of composite laminates using linearized contact law," Composite Structures, vol. 66, pp. 125-132, 2004.

[12] K. Iimura, S. Watanabe, M. Suzuki, M. Hirota, and K. Higashitani, "Simulation of entrainment of agglomerates from plate surfaces by shear flows," Chemical Engineering Science, vol. 64, pp. 1455-1461, 2009.

[13] D. Hays, "Electrostatic adhesion of non-uniformly charged dielectric sphere," Int. Phys. Conf. ser., vol. 118, p. 223228, 1991.

[14] - Role of Electrostatics in Adhesion, in Fundamentals of Adhesion, L.-H. Lee, Ed. PLENUM PRESS, 1991.

[15] M. Krstić, I. Kanellakopoulos, and P. Kokotović, "Adaptive nonlinear control without overparametrization," Systems and Control Letters, vol. 19, pp. 177-185, 1992.

[16] J. Gauthier, H. Hammouri, and S. Othman, "A simple observer for nonlinear systems. application to bioreactors." ITAC, vol. 37, no. 6 , pp. 875-880, 1992. 